\title{
Outcomes of COVID-19 in Solid Organ Transplants
}

Saritha Ranabothu ${ }^{1}$, Swetha Rani Kanduri ${ }^{2}$, Krishna Nalleballe ${ }^{3}$, Wisit Cheungpasitporn ${ }^{4}$, Sanjeeva Onteddu $^{3}$, Karthik Kovvuru ${ }^{2}$

1. Pediatrics, University of Arkansas for Medical Sciences, Little Rock, USA 2. Nephrology, Ochsner Medical Center, New Orleans, USA 3. Neurology, University of Arkansas for Medical Sciences, Little Rock, USA 4. Nephrology, University of Mississippi Medical Center, Jackson, USA

Corresponding author: Karthik Kovvuru, karthikreddy.999@gmail.com

\begin{abstract}
The novel coronavirus disease 2019 (COVID-19) is a global pandemic affecting millions of people worldwide. Solid organ transplant (SOT) recipients are probably at higher risk of severe infection and associated complications from COVID-19. Data on clinical outcomes of COVID-19 infection in SOT recipients are limited. Using the TriNetX database, patients with laboratory-confirmed COVID-19 from January 20, 2020, to July 7,2020 , were included in the study. We compared clinical outcomes comprising hospitalization, need for critical care services, intubation, and mortality among SOT recipients and patients without SOT. Of 30,573 laboratory-confirmed COVID-19 patients, 288 had SOT. Patients with SOT were more likely to be hospitalized (37.2\% vs. $12.2 \%$; $<$ 0.0001), needed critical care services (6.9\% vs. $2.3 \%$; $<<0.0001)$, needed intubation (7.9\% vs. $2.0 \%$; $<<0.0001)$, and had a higher 30-day mortality ( $11.1 \%$ vs. $3.8 \%$; $p<0.0001)$. Patients in the transplant group were older (55.4 vs. 47.6 years; $p<0.0001$ ) and had a higher prevalence of medical co-morbidities. SOT recipients are at significant risk of adverse COVID-19 related outcomes, including hospitalization, need for critical care services, and 30-day mortality, likely due to multiple comorbid conditions.
\end{abstract}

Categories: Internal Medicine, Infectious Disease, Transplantation

Keywords: covid 19, patient outcomes, solid organ transplant, transplant

\section{Introduction}

The ongoing pandemic of the novel coronavirus disease 2019 (COVID-19) infection has implied a tremendous health hazard on the world population. So far, approximately 13 million people are infected with COVID-19, leading to 565,000 reported deaths and we continue to face significant challenges [1]. COVID-19 infection caused by SARS-CoV-2 (severe acute respiratory syndrome coronavirus 2) has not only posed tremendous mortality and morbidity risk but also impacted financial, social, and emotional aspects of human well-being [1]. Solid organ transplant (SOT) recipients presumably are at high risk for COVID-19 infection, especially elderly SOT recipients aged > 60 years [2,3]. Low threshold for testing COVID-19 infection in transplant population is probably beneficial as they manifest atypical symptoms, predominantly diarrhea [4]. The spectrum of clinical disease varies from asymptomatic viral infection to life-threatening illness necessitating mechanical ventilation and renal replacement therapies [2,5]. COVID-19 infection is associated with elevated levels of cytokines, particularly IL-6 (interleukin-6), from an exaggerated host immune response [6,7]. In determining the course of disease, cellular immunity plays a notable role, placing SOT recipients at potential risk of severe complications from COVID-19 infections [8].

Earlier reports suggested similar clinical courses and favorable outcomes in SOT recipients compared with non-SOT population, likely secondary to attenuated immune and cytokine responses [9-11]. Heterogeneous clinical course among SOT cohort, with potential of rapid progression to complicated and fatal clinical course of acute respiratory distress syndrome, has also been reported [12]. Recent studies have reported severe clinical course and worse outcomes while on immunosuppression, with associated co-morbidities $[4,13-14]$. Given the disparities in existing data, we conducted this study to compare the outcomes among SOT recipients and non-SOT population with COVID -19 infection.

\section{Materials And Methods}

TriNetX, a world health collaborative clinical research database that facilitates collecting real-time electronic medical record data from the network of health care organizations across the United States and internationally, was used for extracting information about de-identified COVID -19 patients with and without SOT. "COVID-19 Research Network" in TriNetX represents an extremely large, worldwide COVID19 database.

While the TriNetX database does not acknowledge data downloads or individual patient information for review, it authorizes analysis in the form of queries. Queries made through the TriNetX database operate through browser and real-time features. Several studies on COVID - 19 were published to date using the 
TriNetX database [15-19] and have been described in detail elsewhere [20]. At the University of Arkansas for Medical Sciences, Arkansas Clinical Data Repository and Department of Biomedical Informatics manage TriNetX data.

\section{Study protocol}

Study protocol was implemented after appropriate approval was obtained from the Institutional Review Board. The analysis was conducted on July 7, 2020, on the TriNetX COVID-19 Research Network. We identified SOT patients using the following ICD-10 (the International Classification of Diseases, Tenth Revision) codes: kidney transplant (Z94.0), heart transplant (Z94.1), lung transplant (Z94.2), and liver transplant (Z94.4) [21]. Patients with laboratory-confirmed COVID-19 on or after January 20, 2020, to July 7, 2020, were identified using the LOINC (Logical Observation Identifiers Names and Codes) code (9088: SARS coronavirus 2 and related RNA positive). Thereafter, baseline demographics, co-morbidities, and clinical outcomes, including hospitalization, intubation, need for critical care services, and mortality within 30 days from diagnosis of COVID-19, were compared in patients with and without SOT. TriNetX analytics function was utilized in performing statistical analysis. Outcomes were analyzed using Z-test, propensity score matching (demographics and co-morbidities) was conducted using a t-test. Descriptive statistics were reported as number of observations and percentage or as mean \pm standard deviation, as applicable. Two physicians (K. N. and S. O.) independently performed these queries.

\section{Results}

A total of 288 SOT patients were identified using the TriNetX database, with laboratory-confirmed COVID19 between January 20, 2020, and July 7, 2020. Among them, 109 (37.8\%) patients were females, 89 (30.9\%) patients were Caucasians, 83 (28.8\%) African-Americans, 37 (12.8\%) were Hispanic, and 79 (27.4\%) were of other races/ethnicities/unknown. There were 30,285 laboratory-confirmed COVID-19 patients without SOT in the database. Baseline characteristics such as race, gender, and co-morbidities in the transplant versus non-transplant groups are detailed in Table 1 . 


\section{Cureus}

\begin{tabular}{|c|c|c|c|}
\hline & SOT patients & Non-SOT patients & p-Value \\
\hline Number of patients & 288 & 30,285 & NA \\
\hline Age (years) & $55.4 \pm 15$ & $47.6 \pm 20.3$ & $<0.0001$ \\
\hline Female & 109 (37.8\%) & 15,913 (52.5\%) & $<0.0001$ \\
\hline \multicolumn{4}{|l|}{ Race } \\
\hline Caucasian & 89 (30.9\%) & 11,187 (36.9\%) & 0.0346 \\
\hline African American & $83(28.8 \%)$ & $5,715(18.8 \%)$ & $<0.0001$ \\
\hline Hispanic (ethnicity) & $37(12.8 \%)$ & $4,566(15.1 \%)$ & 0.2923 \\
\hline \multicolumn{4}{|l|}{ Co-morbid conditions } \\
\hline Hypertension & $262(90.9 \%)$ & $7,236(23.9 \%)$ & $<0.0001$ \\
\hline Diabetes mellitus & $164(56.9 \%)$ & $3,841(12.7 \%)$ & $<0.0001$ \\
\hline Ischemic heart disease & $134(46.5 \%)$ & $2,147(7.1 \%)$ & $<0.0001$ \\
\hline Chronic kidney disease & $237(82.3 \%)$ & $1,516(5.0 \%)$ & $<0.0001$ \\
\hline Heart failure & $110(38.2 \%)$ & $1,527(5.0 \%)$ & $<0.0001$ \\
\hline Atrial fibrillation and flutter & 64 (22.2\%) & $1,194(3.9 \%)$ & $<0.0001$ \\
\hline Obstructive pulmonary disease & $46(15.9 \%)$ & $1,149(3.8 \%)$ & $<0.0001$ \\
\hline Overweight and obesity & $114(39.6 \%)$ & $4,358(14.4 \%)$ & $<0.0001$ \\
\hline Nicotine dependence & $41(14.2 \%)$ & $1,873(6.2 \%)$ & $<0.0001$ \\
\hline Ischemic stroke & $16(5.6 \%)$ & $690(2.3 \%)$ & 0.0002 \\
\hline
\end{tabular}

TABLE 1: Baseline demographics and co-morbidities of SOT and non-SOT patients with COVID-

19.

SOT, solid organ transplant

Among the two groups, patients with transplant were older (55.4 vs. 47.6 years; $\mathrm{p}<0.0001$ ) and had a significantly higher prevalence of co-morbidities including diabetes mellitus, ischemic heart disease, hypertension, smoking, obesity, chronic kidney disease, chronic obstructive pulmonary disease, heart failure, atrial fibrillation, and prior ischemic stroke $(\mathrm{p}<0.0001$ except for ischemic stroke for which $\mathrm{p}=$ 0.0002) (Table 1). When comparing outcomes, analysis showed statistically significant higher need for hospitalization (37.2\% vs. $12.2 \%$; $<$ < 0.0001 ) critical care services (6.9\% vs. $2.3 \%$; $<<0.0001)$, intubation (7.9\% vs. $2.0 \%$, p < 0.0001), and higher 30 -day mortality $(11.1 \%$ vs. $3.8 \%$; $\mathrm{p}<0.0001)$ in the SOT group (Table 2). 


\section{Cureus}

\begin{tabular}{|c|c|c|c|c|}
\hline & SOT patients & Non-SOT patients & p-Value & Odds ratio \\
\hline Number of patients & 288 & 30,285 & NA & NA \\
\hline \multicolumn{5}{|l|}{ Clinical outcome } \\
\hline Hospitalization & $107(37.2 \%)$ & $3,698(12.2 \%)$ & $<0.0001$ & $4.25(3.34-5.41)$ \\
\hline Critical care services & $20(6.9 \%)$ & $690(2.3 \%)$ & $<0.0001$ & $3.20(2.02-5.07)$ \\
\hline Intubation & $23(7.9 \%)$ & $621(2.0 \%)$ & $<0.0001$ & $4.15(2.69-6.39)$ \\
\hline Mortality & $32(11.1 \%)$ & $1,155(3.8 \%)$ & $<0.0001$ & $3.15(2.17-4.57)$ \\
\hline
\end{tabular}

TABLE 2: Comparison of outcomes among SOT and non-SOT patients with COVID-19.

Note: $95 \%$ confidence interval provided within bracket for each odds ratio.

SOT, solid organ transplant; NA, not applicable

Among the 288 SOT patients, 224 were kidney transplant patients. In these 224 patients, 88 (39.3\%) patients needed hospitalization, 15 (6.7\%) patients needed critical care services, 20 (8.9\%) patients needed intubation, and 30-day mortality was seen in 21 (9.4\%) patients (Table 3). As a small number of patients with liver, lung, and heart transplant acquired COVID-19, meaningful conclusions regarding outcomes could not be ascertained.

\begin{tabular}{|l|l|}
\hline Number of patients & Kidney transplant patients \\
\hline Clinical outcome & 224 \\
\hline Hospitalization & $88(39.3 \%)$ \\
\hline Critical care services & $15(6.7 \%)$ \\
\hline Intubation & $20(8.9 \%)$ \\
\hline 30 day- mortality & $21(9.4 \%)$ \\
\hline
\end{tabular}

TABLE 3: Subgroup analysis of outcomes among COVID-19 infected kidney transplant patients.

Among these 288 patients, 61 (21\%) were from North East, 62 (22\%) from Midwest, 56 (19\%) from West, 46 (16\%) from South of the US, and 63 (22\%) from outside the US.

\section{Discussion}

Current literature on SOT recipients with COVID infection is dynamic and rapidly evolving. Our study is unique and one of the largest reported thus far, and includes 288 SOT recipients with COVID infection compared with 30,285 COVID positive non-SOT population. While some early small case series reported favorable outcomes among SOT recipients during their initial clinical course [12,22], large studies with longer follow-ups reported greater adverse events [14,23]. In the study by Pereira et al. [14], which included 90 SOT recipients with COVID-19 infection in New York City during a three-week period, $76 \%$ of patients were hospitalized and $34 \%$ required ICU, with $18 \%$ overall mortality (52\% ICU mortality). A recent international study among COVID infected kidney transplant recipients, with a 52-day mean follow-up, reported $32 \%$ mortality among hospitalized patients and $52 \%$ mortality among patients in ICU, with a median time to death of 15 days [23]. As discussed in the Results section, our study reaffirms that COVID like any other infection causes adverse outcomes in SOT recipients and that the earlier thoughts of attenuated immune response potentially offering protection from cytokine storm is probably not true. Our results, similar to recently published reports by Fernández-Ruiz et al. [4] and Akalin et al. [13], emphasize that associated co-morbidities of SOT recipients probably contribute to increased vulnerability, as well as unpredictable and fatal clinical course.

There are some intrinsic limitations to our study, and some of them are inherent to large databases. First, 
this was a retrospective study and the data were obtained based on ICD 10-coding, which could be subjected to reporting bias. Second, it remains unclear whether adverse outcomes are secondary to severe COVID infection versus an effect of organ dysfunction among transplant patients. Third, subgroup analysis and outcomes among patients with heart, liver, and lung transplant could not be provided given the very limited data to make any analysis. Last, participants could have false-positive results on how COVID-19 is tested, and such limitations exist in the majority of reports on COVID-19 during an ongoing pandemic.

\section{Conclusions}

Our study depicted worse outcomes with an increased need for hospitalization among COVID-19 infected SOT recipients when compared with COVID-19 infected non-SOT population likely due to the prevalence of multiple co-morbid conditions in SOT recipients, suggesting that SOT recipients should receive utmost care to help prevent this dreadful infection and early referral to transplant centers with a higher level of care. Spreading awareness and education regarding preventive measures against COVID-19 infection are crucial in providing protection to these immunologically challenged populations. Implementing telehealth might help to curb some exposure risk.

\section{Additional Information \\ Disclosures}

Human subjects: Consent was obtained by all participants in this study. University of Arkansas for Medical Center issued approval 261137. Local IRB deemed this study to be "not human subject research" (global deidentified covid-19 research network data designated for research use) and no IRB approval was needed. Animal subjects: All authors have confirmed that this study did not involve animal subjects or tissue. Conflicts of interest: In compliance with the ICMJE uniform disclosure form, all authors declare the following: Payment/services info: All authors have declared that no financial support was received from any organization for the submitted work. Financial relationships: All authors have declared that they have no financial relationships at present or within the previous three years with any organizations that might have an interest in the submitted work. Other relationships: All authors have declared that there are no other relationships or activities that could appear to have influenced the submitted work.

\section{References}

1. Fung M, Chiu CY, DeVoe C, et al.: Clinical outcomes and serologic response in solid organ transplant recipients with COVID- 19: a case series from the United States [Online ahead of print]. Am J Transplant. 2020, 10.1111/ajt.16079

2. Zhou F, Yu T, Du R, et al.: Clinical course and risk factors for mortality of adult inpatients with COVID-19 in Wuhan, China: a retrospective cohort study. Lancet. 2020, 395:1054-1062. 10.1016/s0140-6736(20)305663

3. Nacif LS, Zanini LY, Waisberg DR, Pinheiro RS, Galvão F, Andraus W, D'Albuquerque LC: COVID-19 in solid organ transplantation patients: a systematic review. Clinics (Sao Paulo). 2020, 75:1983-1983. 10.6061/clinics/2020/e1983

4. Fernández-Ruiz M, Andrés A, Loinaz C, et al.: COVID-19 in solid organ transplant recipients: a single-center case series from Spain. Am J Transplant. 2020, 20:1849-1858. 10.1111/ajt.15929

5. Wu Z, McGoogan JM: Characteristics of and important lessons from the coronavirus disease 2019 (COVID19) outbreak in China: summary of a report of 72314 cases from the Chinese Center for Disease Control and Prevention. JAMA. 2020, 323:1239-1242. 10.1001/jama.2020.2648

6. Batlle D, Soler MJ, Sparks MA, et al.: Acute kidney injury in COVID- 19: emerging evidence of a distinct pathophysiology. J Am Soc Nephrol. 2020, 31:1380-1383. 10.1681/asn.2020040419

7. Delvaeye M, Conway EM: Coagulation and innate immune responses: can we view them separately? . Blood. 2009, 114:2367-2374. 10.1182/blood-2009-05-199208

8. Kronbichler A, Gauckler P, Windpessl M, Il Shin J, Jha V, Rovin BH, Oberbauer R: COVID- 19: implications for immunosuppression in kidney disease and transplantation. Nat Rev Nephrol. 2020, 16:365-367. 10.1038/s41581-020-0305-6

9. Zhu L, Gong N, Liu B, et al.: Coronavirus disease 2019 pneumonia in immunosuppressed renal transplant recipients: a summary of 10 confirmed cases in Wuhan, China. Eur Urol. 2020, 77:748-754. 10.1016/j.eururo.2020.03.039

10. Columbia University Kidney Transplant Program: Early description of coronavirus 2019 disease in kidney transplant recipients in New York. J Am Soc Nephrol. 2020, 31:1150-1156. 10.1681/asn.2020030375

11. Ning L, Liu L, Li W, et al.: Novel coronavirus (SARS-CoV-2) infection in a renal transplant recipient: case report. Am J Transplant. 2020, 20:1864-1868. 10.1111/ajt.15897

12. Bhoori S, Rossi RE, Citterio D, Mazzaferro V: COVID-19 in long-term liver transplant patients: preliminary experience from an Italian transplant centre in Lombardy. Lancet Gastroenterol Hepatol. 2020, 5:532-533. 10.1016/s2468-1253(20)30116-3

13. Akalin E, Azzi Y, Bartash R, et al.: Covid-19 and kidney transplantation. N Engl J Med. 2020, 382:2475-2477. 10.1056/NEJMc2011117

14. Pereira MR, Mohan S, Cohen DJ, et al.: COVID-19 in solid organ transplant recipients: Initial report from the US epicenter. Am J Transplant. 2020, 20:1800-1808. 10.1111/ajt.15941

15. TriNetX. (2020). Accessed: September 1, 2020: http://Www.Trinetx.com.

16. Nalleballe K, Reddy Onteddu S, Sharma R, et al.: Spectrum of neuropsychiatric manifestations in COVID-19. Brain Behav Immun. 2020, 88:71-74. 10.1016/j.bbi.2020.06.020

17. Onteddu SR, Nalleballe K, Sharma R, Brown AT: Underutilization of health care for strokes during the 


\section{Cureus}

COVID-19 outbreak. Int J Stroke. 2020, 15:9-10. 10.1177/1747493020934362

18. Ranabothu S, Onteddu S, Nalleballe K, Dandu V, Veerapaneni K, Veerapandiyan A: Spectrum of COVID-19 in children. Acta Paediatr. 2020, 109:1899-1900. 10.1111/apa.15412

19. Banerjee D, Popoola J, Shah S, Ster IC, Quan V, Phanish M: COVID-19 infection in kidney transplant recipients. Kidney Int. 2020, 97:1076-1082. 10.1016/j.kint.2020.03.018

20. Shah VA, Nalleballe K, Zaghlouleh E, Onteddu S: Acute encephalopathy is associated with worse outcomes in COVID-19 patients. Brain Behav Immun Health. 2020, 8:100136.

21. International Classification of Diseases, Tenth Revision, Clinical Modification (ICD-10-CM). (2020). Accessed: September 1, 2020: https://www.cdc.gov/nchs/icd/icd10cm.htm.

22. Zhong Z, Zhang Q, Xia H, et al.: Clinical characteristics and immunosuppressant management of coronavirus disease 2019 in solid organ transplant recipients. Am J Transplant. 2020, 20:1916-1921. 10.1111/ajt.15928

23. Cravedi P, Suraj SM, Azzi Y, et al.: COVID-19 and kidney transplantation: results from the TANGO International Transplant Consortium [Online ahead of print]. Am J Transplant. 2020, 10.1111/ajt.16185 